\title{
A viral infection explanation for Kawasaki disease in general and for COVID-19 virus-related Kawasaki disease symptoms
}

\author{
Kevin $\operatorname{Roe}^{1}$ D
}

Received: 31 May 2020 / Accepted: 28 June 2020 / Published online: 7 July 2020

(c) Springer Nature Switzerland AG 2020

\begin{abstract}
SARS-CoV-2, a new virus that appeared in Wuhan, China, in 2019 has approximately an $80 \%$ genomic match to the Severe Acute Respiratory Symptom (SARS) virus, which is known to come from a bat virus. Symptoms of Kawasaki disease in general and incomplete Kawasaki disease have been seen in a subset of pediatric patients having a current or previous infection of SARS-CoV-2. A viral infection, such as a SARS-CoV-2 virus infection, could result in extensive antigen-antibody immune complexes that cannot be quickly cleared in a subset of patients and thus create a type III hypersensitivity immune reaction and cause Kawasaki disease or Kawasaki disease symptoms (also known as multisystem inflammatory syndrome) in a subset of patients. Extensive binding of antibodies to viral antigens can create antigen-antibody immune complexes, which, if not eliminated in certain individuals having dysfunctional complement systems, can start inflammatory type III hypersensitivity symptoms, including protease releases that can disrupt epithelium, mesothelium, and endothelium basement membranes, and induce pervasive inflammation throughout the body. This could continue after SARS-CoV-2 infections end if the first wave of protease attacks on basement membranes created new secondary autoantibodies and new uncleared antigen-antibody immune complexes.
\end{abstract}

Keywords Kawasaki disease $\cdot$ Emerging diseases $\cdot$ Virus $\cdot$ Immune system $\cdot$ Zoonosis $\cdot$ Inflammation

SARS-CoV-2 virus is a new virus that first appeared in 2019, in Wuhan, China, frequently causing fevers, coughs, diarrhea, fatigue, and numerous deaths (Mao et al. 2020). The SARS-CoV-2 virus matches approximately $80 \%$ of the genome of the Severe Acute Respiratory Symptom (SARS) virus, which originated from a bat virus (Ye et al. 2020). Symptoms of Kawasaki disease in general or incomplete Kawasaki disease have been seen in some pediatric patients having a current or previous SARS-CoV-2 infection (Jankowicz 2020; Jones et al. 2020; McCrindle et al. 2017; Maggio and Corsello 2015). Kawasaki disease in general, incomplete (atypical) Kawasaki disease and Kawasaki disease symptoms (also known as multisystem inflammatory syndrome) seen in some pediatric patients after SARS-CoV-2 viral infections can be the result of virus infections having high replication rates, such as SARS-CoV-2 virus, creating

Kevin Roe: Retired.

Kevin Roe

kevin.roe@att.net

1 San Jose, CA, USA uncleared antigen-antibody immune complexes and type III hypersensitivity immune reaction symptoms, as further discussed below.

The most dangerous Kawasaki disease symptoms include coronary artery inflammation and frequent coronary artery aneurysms. Other Kawasaki disease symptoms include systemic inflammation of arteries, organs, and tissues, which can cause several symptoms: hepatitis-liver inflammation frequently with abdominal pain; lung interstitial pneumonitis-tissue scarring surrounding the lung alveoli; gastrointestinal tract—abdominal pain, vomiting, and diarrhea; aseptic meningitis - brain membrane inflammations; myocarditis - heart muscle inflammation; pericarditis-heart tissue sac inflammation; valvulitis-heart valve inflammation; urinary tract pyuria-urinary pus; pancreatitis-pancreatic inflammation; and lymph-node enlargement (McCrindle et al. 2017).

Kawasaki disease is diagnosed after 5 or more days of fever and the presence of at least four of the five principal symptoms: (1) erythema (skin redness), cracking of lips and a strawberry tongue; (2) a dry conjunctivitis; (3) rashes; (4) erythema and edema (swelling) of hands and feet; and (5) 
multiple cervical neck lymph-node enlargements (McCrindle et al. 2017). If four or more of these principal symptoms appear, particularly with redness and swelling of the hands and feet, a diagnosis requires only 4 days of fever, but the principal symptoms may not all be simultaneously seen (McCrindle et al. 2017). Incomplete Kawasaki disease shows fewer symptoms (Maggio and Corsello 2015). Similarly, some symptoms may moderate in patients after 1-2 weeks of fever, which is typically remittent and spiking (more than $39-40{ }^{\circ} \mathrm{C}$ ); and without appropriate treatment, fever can continue for 1-3 weeks (McCrindle et al. 2017). However, a quick termination of fever after 7 days does not exclude Kawasaki disease (McCrindle et al. 2017).

There are distinctive Kawasaki disease symptoms in the hands and feet, including erythema of palm and soles with painful hardening of the hands or feet often occurring; and finger and toe desquamation (outer skin layer shedding) usually begins within $2-3$ weeks after fever onset, possibly including the palms and soles (McCrindle et al. 2017).

An erythematous rash (red skin from inflammation) typically appears within 5 days from the start of fever; and this is usually a diffuse maculo-papular rash (raised lesions are frequent in pediatric viral infections), with a red skin rash with bumps and erythema multiforme-like rashes (also seen in IgM immune complex depositions); and the rash is typically widespread on the trunk, extremities, and groin with an early desquamation (McCrindle et al. 2017).

Conjunctivitis (pink in the white sclera of the eyes) is a frequent inflammation symptom resulting from viral or bacteria infections, and usually begins after the fever; and anterior uveitis (inflammation of the middle layer of the eyes) is another symptom often seen during the first week of fever (McCrindle et al. 2017).

Symptoms seen on the lips and oral cavity include (1) erythema (skin redness), dryness, peeling, fissuring, cracking, and bleeding of the lips; (2) a "strawberry tongue," with erythema and noticeable mushroom-shaped papillae; and (3) widespread erythema in the oropharyngeal mucosa (McCrindle et al. 2017). Enlarged lymph nodes are the less often seen principal symptom. Lymph-node swelling is usually on one side of the neck, with diameters equal to or greater than $1.5 \mathrm{~cm}$ (McCrindle et al. 2017).

It should be noted that most Kawasaki disease symptoms previously listed can come from a pervasive and systemic inflammation created by a type III hypersensitivity reaction of the immune system (Agarwal et al. 1995; Punt et al. 2019). Below is a discussion of how type III hypersensitivity reactions could result from some viruses that infect a large surface area of tissue with a high replication rate, such as SARS-CoV-2.

Several bat viruses have high replication rates in infected cells after transmission to secondary species, such as viruses that evolved their replication traits during their infections of bats and thus had their viral replication processes sculpted by bat immune system responses (Brook et al. 2020). A discussion of why bat viruses can replicate quickly in human cells is provided in another paper (Roe 2020).

Individual immune systems can differ by age, health, and genome in their production speed and quantity of interferons, NK cells, T cells, and antibodies that they will mobilize against bat virus infections. A feeble antiviral NK-cell and T-cell response to SARS-CoV-2 has been widely seen in several patients (Pavlovich et al. 2018; Zhang et al. 2020). Antibodies will be the remaining antiviral defense (Jazayeri and Poh 2019). The main step would be antibodies from B-2 or B-1 cells (mostly IgM, with some IgG or IgA antibodies) targeting viral antigens and making antigen-antibody immune complexes (Agarwal et al. 1995; Punt et al. 2019). Antigen-antibody immune complexes are tagged for phagocytic elimination by a normal complement system and asymptomatically cleared by phagocytes in a normal spleen or liver, but if an individual's immune system cannot quickly eliminate them, they can induce several type III hypersensitivity symptoms, including fever, inflammation, microvascular thrombosis (microvascular blood clots), glomerulonephritis, vasculitis, rashes, vascular purpura, joint pain, etc. (Agarwal et al. 1995; Punt et al. 2019).

Uncleared antigen-antibody immune complexes can activate receptors of mast cells, neutrophils, and macrophages, initiating the release of several inflammatory cytokines and increasing blood vessel permeability, allowing antigen-antibody immune complexes to pass through capillary walls to deposit in tissues (Punt et al. 2019). This creates tissue inflammation and activates the complement system, resulting in releases of anaphylatoxin chemokines C3a and C5a that will attract neutrophils and macrophages for degranulation to secrete more proinflammatory chemokines, cytokines, proteases, and prostaglandins (Roe 2020). Proteases can destroy epithelium, mesothelium, and endothelium basement membrane proteins including elastin and collagen, essential to lungs and blood vessels throughout the body's organs, skin and other luminal tissues, and even attack cartilage, causing joint pain (Punt et al. 2019). Symptoms of antigen-antibody immune complex induced inflammation have appeared in several patients infected by SARS-CoV-2, such as inflammatory cytokines, vasculitis, microvascular thrombosis, and spleen and lymph-node destruction (Zhang et al. 2020). A summary of the steps creating Kawasaki disease symptoms is shown in Table 1.

In some cases, the first wave of protease attacks on the epithelium, mesothelium, and endothelium basement membranes could result in new immunogenic antigens, which can eventually create new autoantibodies (McAdoo and Pusey 2017). These autoantibodies could create secondary antigen-antibody immune complexes that, if uncleared, due to inadequacies of the spleen, liver, or complement system, 
Table 1 Summary of the steps creating Kawasaki disease symptoms

1. Infection of host with a high replication rate virus, such as SARS-CoV-2

2. Failure of the innate immune system to control the virus replication

3. Adaptive immune system produces antibodies to neutralize the virus

4. Large numbers of antigen-antibody immune complexes are created

5. Host is unable to quickly eliminate antigen-antibody immune complexes

6. Antigen-antibody immune complexes activate receptors of mast cells, neutrophils, and macrophages, initiating release of inflammatory cytokines and increasing blood vessel permeability - this is the beginning of a Type III hypersensitivity reaction

7. Antigen-antibody immune complexes deposit in capillary tissues, causing inflammation

8. Tissue inflammation activates the complement system, resulting in releases of anaphylatoxin chemokines C3a and C5a

9. C3a and C5a attract neutrophils and macrophages for degranulation to secrete more proinflammatory chemokines, cytokines, proteases, and prostaglandins

10. Proteases destroy epithelium, mesothelium, and endothelium basement membrane proteins including collagen and elastin, essential to lungs and blood vessels throughout the body's organs, skin and other luminal tissues, and attack cartilage, causing joint pain

would result in secondary type III hypersensitivity immune reactions and Kawasaki disease symptoms after the original SARS-CoV-2 infection had ended (Bajic et al. 2015; Punt et al. 2019).

Antigen-antibody immune complexes and eventual protease release can result from antibodies targeting viral antigens created during the viral budding stage of endothelial cells of blood vessels infected by SARS-CoV-2 virus; therefore, viral infections of endothelial cells could contribute to a type III hypersenstivity reaction and Kawasaki disease symptoms in a subset of pediatric patients having SARSCoV-2 (Punt et al. 2019; Zhang et al. 2020; Roe 2020).

In conclusion, a virus that can infect a large surface area of tissue with a high replication rate, similar to SARSCoV-2, can create extensive antigen-antibody immune complexes that cannot be quickly eliminated (phagocytized) in a subset of immunodeficient patients and, thus, create a type III hypersensitivity immune reaction and cause Kawasaki disease; or cause Kawasaki disease symptoms in a subset of patients infected with SARS-CoV-2 virus. This can include protease releases that can disrupt luminal tissue epithelium, mesothelium, and endothelium basement membranes, and destroy elastin, collagen, and cartilage, and create pervasive inflammation throughout the body. Kawasaki disease, incomplete Kawasaki disease, or Kawasaki disease symptoms can result in some pediatric patients being unable to avoid a type III hypersensitivity reaction. This could continue after SARS-CoV-2 infections if the first wave of protease attacks on basement membranes created new autoantibodies and new uncleared antigen-antibody immune complexes. In summary, Kawasaki disease results if some pediatric patients have certain virus infections and have immunodeficiencies with slow or weak mobilizations of interferons, NK cells, and T cells to suppress viral replication, and thus depend on antibodies, while lacking the ability to quickly eliminate the extensive antigen-antibody immune complexes that are created.
Funding This research did not receive any specific grant from funding agencies in the public, commercial, or not-for-profit sectors.

\section{Compliance with ethical standards}

Conflict of interest The author has no potential conflicts of interest.

Ethics Statement The author confirms that the ethical policies of the journal, as noted on the journal's author guidelines page, have been adhered to. No ethical approval was required as this is a review article with no original research data.

Data Availability Statement Data sharing is not applicable to this article as no new data were created or analyzed in this study.

\section{References}

Agarwal SK, Ghosh PK, Gupta D (1995) Cardiac surgery and coldreactive proteins. Ann Thorac Surg 60:1143-1150

Bajic G, Degn SE, Thiel S, Andersen GR (2015) Complement activation, regulation, and molecular basis for complement-related diseases. EMBO J 34(22):2735-2757

Brook CE, Boots M, Chandran K, Dobson AP, Drosten C, Graham AL, Grenfell BT et al (2020) Accelerated viral dynamics in bat cell lines, with implications for zoonotic emergence. eLife 9:e48401. https://doi.org/10.7554/eLife.48401

Jankowicz M (2020) Doctors in Italy have found a link between the coronavirus and the rare inflammatory disease seen in more than 100 children. Business Insider. https://news.yahoo.com/doctorsitaly-found-between-coronavirus-124700554.html

Jazayeri SD, Poh CL (2019) Development of universal influenza vaccines targeting conserved viral proteins. Vaccines 7(4):169. https ://doi.org/10.3390/vaccines7040169

Jones V, Mills M, Suarez D, Hogan C, Yeh D, Segal J (2020) COVID19 and Kawasaki disease: novel virus and novel case. Hosp Pediatr. https://doi.org/10.1542/hpeds.2020-0123

Maggio MC, Corsello G (2015) Atypical and incomplete Kawasaki disease. Ital J Pediatr 41(Suppl 2):A45

Mao L, Jin H, Wang M, Hu Y, Chen S, He Q, Chang J et al (2020) Neurological manifestations of hospitalized patients with coronavirus disease 2019 in Wuhan, China. JAMA Neurol. https://doi. org/10.1001/jamaneurol.2020.1127 
McAdoo SP, Pusey CD (2017) Anti-glomerular basement membrane disease. Clin J Am Soc Nephrol 12(7):1162-1172. https://doi. org/10.2215/CJN.01380217

McCrindle BW, Rowley AH, Newburger JW et al (2017) Diagnosis, treatment, and long-term management of Kawasaki disease. Circulation 135:e927-e999. https://doi.org/10.1161/cir.0000000000 000484

Pavlovich SS, Lovett SP, Koroleva G, Sanchez-Lockhart M, Kepler TB, Palacios G (2018) The Egyptian rousette genome reveals unexpected features of bat antiviral immunity. Cell 173:1098-1110

Punt J, Stranford SA, Jones PP, Owen JA (2019) Allergy, hypersensitivities, and chronic inflammation. Kuby immunology, 8th edn. W. H. Freeman and Company, New York, pp 549-591

Roe K (2020) High COVID-19 virus replication rates, the creation of antigen-antibody immune complexes, and indirect hemagglutination resulting in thrombosis. Transbound Emerg Dis. https://doi.org/10.1111/tbed.13634

Ye G, Pan Z, Pan Y, Deng Q, Chen L, Li J, Li Y, Wang X (2020) Clinical characteristics of severe acute respiratory syndrome coronavirus 2 reactivation. J Infect. https://doi.org/10.1016/j. jinf.2020.03.001

Zhang W et al (2020) The use of anti-inflammatory drugs in the treatment of people with sever coronavirus disease 2019 (COVID19): the perspectives of clinical immunologists from China. Clin Immunol. https://doi.org/10.1016/j.clim.2020.108393

Publisher's Note Springer Nature remains neutral with regard to jurisdictional claims in published maps and institutional affiliations. 